\title{
Integrating Uncertain Knowledge in a Domain Ontology for Room Concept Classifications
}

\author{
Joana Hois \\ I1-[OntoSpace], SFB/TR8 Spatial Cognition, Universität Bremen \\ Bremen, Germany \\ Kerstin Schill \\ A5-[ActionSpace], SFB/TR8 Spatial Cognition, Universität Bremen \\ Bremen, Germany \\ John A. Bateman \\ I1-[OntoSpace], SFB/TR8 Spatial Cognition, Universität Bremen \\ Bremen, Germany
}

\begin{abstract}
Ontologies provide a representation of precise knowledge about concepts, their attributes and relations. The Dempster-Shafer theory provides a representation of epistemic plausibilities. In AI both representations are typically developed separately on purpose, which is appropriate unless their combination is required. Real world applications, however, sometimes require a combination of both.

In this paper we will present such a combination of ontological and uncertain knowledge. Our approach arises from the need of a room classification system for representing room concepts (in the sense of classifying names that are cognitively assigned to rooms, such as 'kitchen', 'laboratory', 'office') that can be derived from objects occurring in the rooms. These room concepts can only be determined with a certain degree of belief, not so much depending on the system's quality as depending on ambiguities in the cognitive assignment of room concepts. Hence, uncertainty about concepts that exist in reality also needs to be represented in the application.
\end{abstract}

\section{Introduction}

Our aim is to develop a system that identifies the room concept of a room on the basis of visual information in order to accomplish further tasks based on this classification. Room concepts (cf. [12]) are distinguished by means of the functional role that is associated with actions that can be performed in such rooms. In turn, the functions and actions depend on several physical objects that have to be in the room. The objects occurring in a room therefore imply 
the room's room concept. For example, a kitchen is a place where you can make coffee, wash dishes, or cook. These activities depend on certain objects, namely interior furniture, equipment, or ingredients. In our example, the activity 'make coffee' depends on a coffee machine, a mug, sugar, milk, water, or similar objects. Hence, the perception of the concepts of a room depend on its contained objects as they indicate possible functional aspects [14] and consequently the functional role, i.e. the room concept itself.

This analysis provides significant opportunities for cognitive, spatially aware systems. For instance, intelligent buildings using this system are able to answer user requests, such as "Where can I find an unoccupied kitchen?" or "Tell me all possible seminar rooms that have at least a video projector and a white board." Or autonomous robots can respond to tasks that refer to special activities they can perform considering the respective room concepts and occurring objects. Both examples also show a strong connection to human-computer interaction, possibly realised with natural language dialogues, which can directly access the concepts described above.

For representing necessary concepts of rooms and objects in our application we decided to apply an ontological knowledge structure by developing a domain ontology. The decision to use an ontology is based upon its advantage for formal representation of objects and their relations and its direct support of re-usability and knowledge sharing [7]. For the latter, our domain ontology is guided by an ontological library for linguistic and cognitive engineering [13], which also offers a solid basis for further development. That linguistic terms also have a strong impact on the types of room concepts and objects confirms our decision for using a domain ontology as it can provide a mapping to a linguistic ontology and can be used within a dialogue system [8].

However, we must also represent degrees of uncertainty for each room concept because of the possibility of one room providing multiple functions and uncertain evidence. Thus, the analysis of a room indicates room concepts with a certain evidence. This is due not to the shortcoming of the system but to the fact that a room scene tends to be compatible with more than just one room concept. For example, when the system recognizes a room full of chairs and tables it may be confronted with a study room, a lecture hall, or a seminar room. Thus, we are usually not able to make distinct definitions of a unique room concept and we specify the evidence for a certain room concept instead. In our work this evidence is represented via the Belief function of the Dempster-Shafer theory [18].

In the following, we will give detailed description of the representation of ontological knowledge within our system as well as the representation of uncertain knowledge, and we will demonstrate a combination of ontological and uncertain knowledge considering our application as example. 


\section{Ontological Knowledge}

Ontologies are nowadays widely used in different applications ranging from the attempt to represent complete general knowledge and commonsense, such as OpenCyc ${ }^{1}$, to the use in particular domains to represent specific concepts, such as Medical Information ${ }^{2}$. [9] specify several principles by which ontologies can be modeled and classified and they give a detailed overview of technical issues and applications using ontologies hitherto. This spread of ontological applications also results from the development of the SemanticWeb [5], which is intended to provide a navigable structure aligned by its semantic concepts.

As we have to represent rooms, their interior objects, and the room concepts they support, we require a taxonomical and partonomical structure: The room concepts can be grouped hierarchically in a taxonomy, whereas interior objects are parts of the respective rooms. This representation is also affected by linguistic expressions of the represented concepts caused by the intended dialog between a user and the system, as we have mentioned with the intelligent building example above. Therefore, the structure has to take into account also the semantics of those expressions corresponding with the room scenes. Further considerations are reasoning strategies that are inherently supported by the structure. As all of these requirements are covered by ontological structures (see [9]) and because of the reasons we gave in the introduction, we decided to develop a domain ontology for representing scenes, objects, and room concepts. We finally have to consider the particular modelling of a domain ontology for our application.

Besides the different ontology principles, the role of an ontology can be outlined as "(i) to set out a consistent and well-specified general modelling scheme which is free of contradiction and from which follows a set of generic properties that necessarily hold over the entities covered and, (ii) to support problem solving and inference within the domain of concern" [3]. Yet a realisation of the first claim is not thus simple. Although there are several guidelines for the design of ontologies (for instance [20]), there are still problems about consistency and coherence throughout various ontologies. Complications usually arise in case of ontology mappings and it becomes difficult to mediate between different ontologies because they are not interchangeable. Hence, the sharing of ontological knowledge becomes complex and ontologies are rather remade than re-used. Recently, different approaches try to provide not just guidance but a systematic frame for designing ontologies. As we do not want to encounter the problems of indiscriminately designed ontologies, our developed ontology for rooms and their objects will be integrated into an ontology framework that already provides a more abstract scope for linguistic and cognitive engineering, namely DOLCE [13].

\footnotetext{
${ }^{1}$ http://www.opencyc.org/

${ }^{2}$ http://www.ifomis.uni-saarland.de/
} 


\subsection{Domain Ontology Modelling}

DOLCE was originally developed as a part of the WonderWeb project ${ }^{3}$ with a strong influence from natural language and human commonsense. The basic categories of DOLCE are Perdurants, Endurants, Qualities, and Abstracts. Perdurants describe entities that unfold in time (e.g., an event of some kind), whereas Endurants describe entities that are wholly present at each point in time (e.g., a cup, a glass, a desk, etc.). Qualities inhere in entities, which means that every entity can have certain qualities, even Qualities themselves. The value of these qualities are expressed by entities of the Abstract concept, called quale.

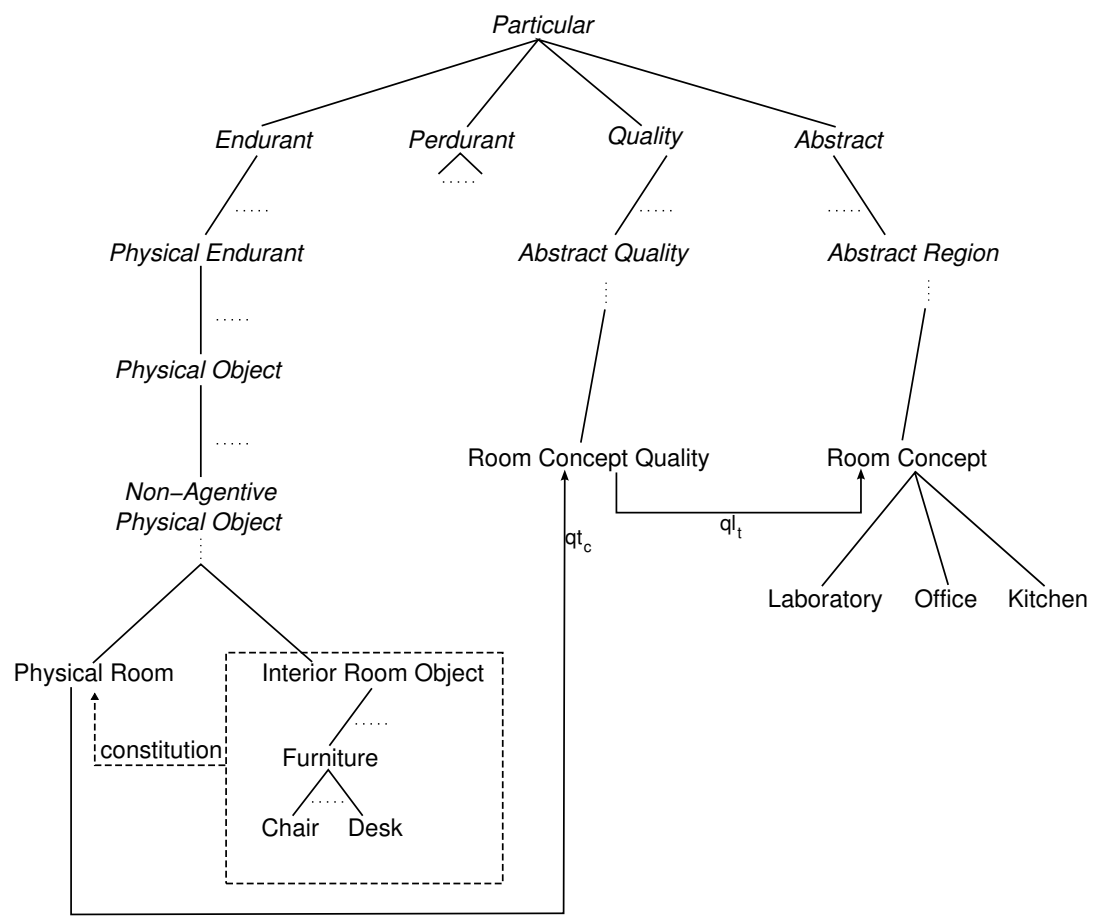

Figure 1: Ontological room concepts of the domain ontology. The concepts in italic are indicating DOLCE's concepts

The rooms we will analyse will be classified as an instance of Non-Agentive Physical Object (a subclass of Endurant) as it defines physical objects that have direct spatial qualities, a life (exist over time), and we do not ascribe intentions, beliefs, or desires to them (in contrast to Agentive Objects). The objects that occur in a room will be an instance of Non-Agentive Physical Object as well because we are currently omitting humans or robots in rooms as their functional indication of the room concept might be low.

${ }^{3} \mathrm{http}$ ///wonderweb.semanticweb.org 
Starting from this we created a hierarchy of specific non-agentive physical objects, to wit physical rooms and their contained objects. Physical rooms in this case define the bare rooms that are characterised by their walls, floors, ceilings, and dimensions and that can be constituted by further physical objects (such as chairs, desks, shelfs, etc.). These physical rooms are not distinguished by their functional role yet. Instead the hierarchy of possible room concepts are classified as an instance of Abstract. This distinction between physical rooms (that contain objects) and the abstract room concepts (that can be represented by a physical room) is quite crucial. It is a precise modeling of the relations between rooms and their room concepts and results in physical rooms having a quality that specifies the room concept it may support owing to its constituting objects (or in fact the functions that the objects are indicating). This physical room quality also resembles the concept of a so-called niche [2, 19]. Figure 1 illustrates the described ontological concepts and their relations. The dashed lines are indicating that there might be other concepts in the hierarchy between DOLCE's concepts and the domain ontology concepts, however, our application will subsume the domain concepts directly under DOLCE's.

This domain ontology design facilitates the representation of our application concern though the supported room concepts of a physical room cannot be classified uniquely even in reality. Imagine a room having a table with chairs surrounded and some pictures on the walls or the like. Without further information it may serve as a dining room, a seminar room, a day room, or all of them as the occurring objects do not give much indication to possible actions that can be performed in this room. In lieu of exactly determining the supported room concepts the application is instead able to indicate them with a certain probability degree. Hence, uncertain factors also need to be represented in our domain ontology. In fact, we integrate belief values of the Dempster-Shafer theory for room concepts' indications. We will introduce this theory in the next section and the integration subsequently.

\section{Dempster-Shafer Theory}

The Dempster-Shafer theory [18] provides the representation and analysis of uncertain knowledge and a method for evidential reasoning. It was originally developed as an enhancement of probabilistic theory, but instead of assigning probabilities to propositions, degrees of belief are assigned and the uncertain knowledge is represented by a Belief function.

Belief can be assigned not only to single propositions but to sets of proposition. All proposition are gathered in the frame of discernment $\Theta$, which is named after its epistemic nature, as it acquires "its meaning from what we know or think we know; the distinctions that it embodies will be embedded within the matrix of our language and its associated conceptual structures and will depend on those structures for whatever accuracy and meaningfulness they possess" [18]. The belief of the smallest subsets of $\Theta$ is generated by the basic probability assignment function 


$$
\mathrm{m}: 2^{\Theta} \rightarrow[0,1] .
$$

To calculate the total belief of a subset of $\Theta$, the basic probabilities of all proper subsets have to be summed in a Belief function

$$
\operatorname{Bel}(A)=\sum_{B \subset A} m(B) .
$$

Different Belief functions that relate to the same frame of discernment but derive from distinct bodies of evidence can be combined by using Dempster's Rule of Combination, which is a belief function based on the combined evidences for computing their orthogonal sum. For the combination of different belief this function $\left(\left(\cdots\left(\mathrm{Bel}_{1} \oplus \mathrm{Bel}_{2}\right) \cdots\right) \oplus \mathrm{Bel}_{n-1}\right) \cdots \mathrm{Bel}_{n}$ combines the belief $\mathrm{Bel}_{1}$, $\ldots, \mathrm{Bel}_{n}$ pairwise. For instance, if we have the result of two analyses from distinct points of view of the room, both indicating that the room supports the room concept "office"; viewpoint A with an evidence of 0.7 , viewpoint B with an evidence of 0.9. Then we can combine both as shown in Table 1. Hence, the total evidence for the room concept "office" is 0.97 (the sum of each supporting evidence). For a more detailed presentation of Dempster's Rule, we refer the reader to [18], chapter $3, \S 1$.

Table 1: Example of using Dempster's Rule of Evidence for combining different evidences for the room concept "office" from viewpoint A and B

\begin{tabular}{ccc}
\hline & A: "Office" $(0.7)$ & A: $\Theta(0.3)$ \\
\hline B: "Office" $(0.9)$ & 0.63 & 0.27 \\
B: $\Theta(0.1)$ & 0.07 & 0.03 \\
\hline
\end{tabular}

A major advantage of the Dempster-Shafer theory is its explicit representation of ignorance [4] as it makes a clear distinction between the lack of knowledge and the assignment of degrees of belief to the negation of a proposition. Hence, there is no need for the belief of a proposition and the belief of its negation to sum up to 1 because remaining propositions will not be affected without explicit assignments. In our application the belief that a physical room may be used as an office for a certain degree does not determine the belief of other possible room concepts, for instance, a study room (especially as the physical room may be used as both room concepts if we employ them in our application). The use of subjective belief of specialists is also a great benefit if no a priori probabilities are available [16]. This provides the possibility of assigning beliefs to physical room concepts depending on occurring objects without the investigation of a vast number of empirically measured physical room data (see for further details the exemplary implementation in section 5).

When applying Dempster's Rule of Combination new belief can be combined with the old one, which results in a belief update. This benefits our system's implementation depending on our application, which analyses one object after another and can therefore calculate current belief distributions iteratively. 
It has been observed that "very little has been done to perform reasoning or inferencing with information represented in terms of belief functions" [4]. Hence, using a domain ontology, which already provides a reasoning strategy in its structure to integrate this information seems to be a promising suggestion.

Although a disadvantage of the Dempster-Shafer theory is its computational complexity, simplification algorithms have been developed $[1,10]$ that restrict the frame of discernment to a hierarchical structure, which is already conformant with an ontological structure.

Besides the incidental relation between the frame of discernment's epistemic slope and our cognitively assigned room concepts that rely on linguistic expressions, the reasons mentioned above confirm the adequacy of using DempsterShafer's Belief functions for the representation of uncertain knowledge for the room concepts in order to integrate them into an ontology. This integration will be discussed in the next section.

\section{Combination of Domain Ontology and Dempster-Shafer Theory}

As we have now illustrated the need for both the representation of ontological knowledge as well as Dempster-Shafer belief, we will introduce our approach to integrate belief values of the Dempster-Shafer theory into our developed domain ontology.

The clear separation between physical rooms and abstract room concepts turns out to be not only a precise modeling of the relations between rooms and their room concepts but also a sophisticated foundation for the combination of an ontology and the Dempster-Shafer theory. This allows us to assign belief values to the quale values of the quality that a physical room supports a room concept. This is illustrated in Figure 2: Room Concept Quality can have more than just one quale, which are in turn provided with an additional degree of evidence of the Dempster-Shafer theory, namely a belief value between 0 and 1 .

When a physical room with its constituting objects is instantiated, the room can have several qualities that relate it to particular room concepts with a specific degree of evidence. In case of new information about the room concepts the evidences can be updated by using Dempsters Rule of Combination ${ }^{4}$.

This domain ontology design is characterised by particular criteria: First, rooms are represented independently from the room concepts they support and they are uniquely distinguished by their physical dimensions and properties. For instance, if the furniture of a room is removed from the room it will still retain its identity, only its room concept qualities may change. Similarly, rooms are constituted by their objects that are positioned in them and their unique character is not depending on them. Second, the constellation of the constituting objects suggests possible functions that can be performed in the rooms

\footnotetext{
${ }^{4}$ Note: For simplicity we are currently just classifying single concepts. Therefore, we can equate the belief for single propositions (basic probability assignment) with sets of propositions (Belief function).
} 


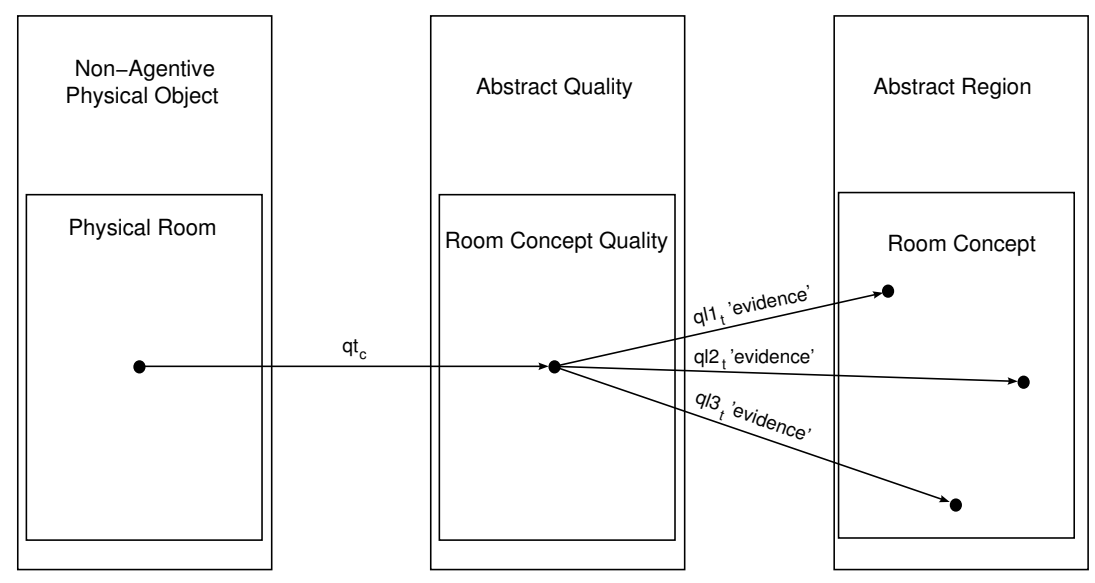

Figure 2: Integrating evidence values into domain ontology

and depending on these functional aspects, possible cognitively assigned room concepts can be derived. Third, the quality that a room may support a certain room concept is specified by a belief value with regard to functions that the constituting objects indicate or not.

Based on this formal representation, we have developed a visual room concept classification system, which is introduced in the next section.

\section{Room Concept Recognition System}

In our application, the room concept classification is incorporated into a cognitive vision system [17] that analyses and classifies an individual object that is displayed in a 2D-image. This hybrid system performs saccadic eye movements on the image and classifies the represented object after performing a supervised learning phase. We have extended this system so that it can analyse possible room concepts of a given input image by reasoning over the extracted and classified individual objects in the image. As we do not want to consider concrete segmentation algorithms yet, we have created exemplary scenes on the basis of real pictures, in which each single object has its own gray value. We also do not allow overlapping or partially hidden objects yet.

The analysis of a complex scene composed of several objects is divided into two processes: On a higher level the system performs eye movements on the whole scene stopping at each fixation point. On a lower level it extracts the object, which is located at each fixation point, from the complete scene and classifies it by performing eye movements solely on this object depending on a prior learning phase. After classifying the single object the system returns to the higher level and resumes eye movement on the complete scene as illustrated in Figure 3. This split between the higher and lower level of eye movements is based on the assumption resulting from experimental results [11, 21] that 


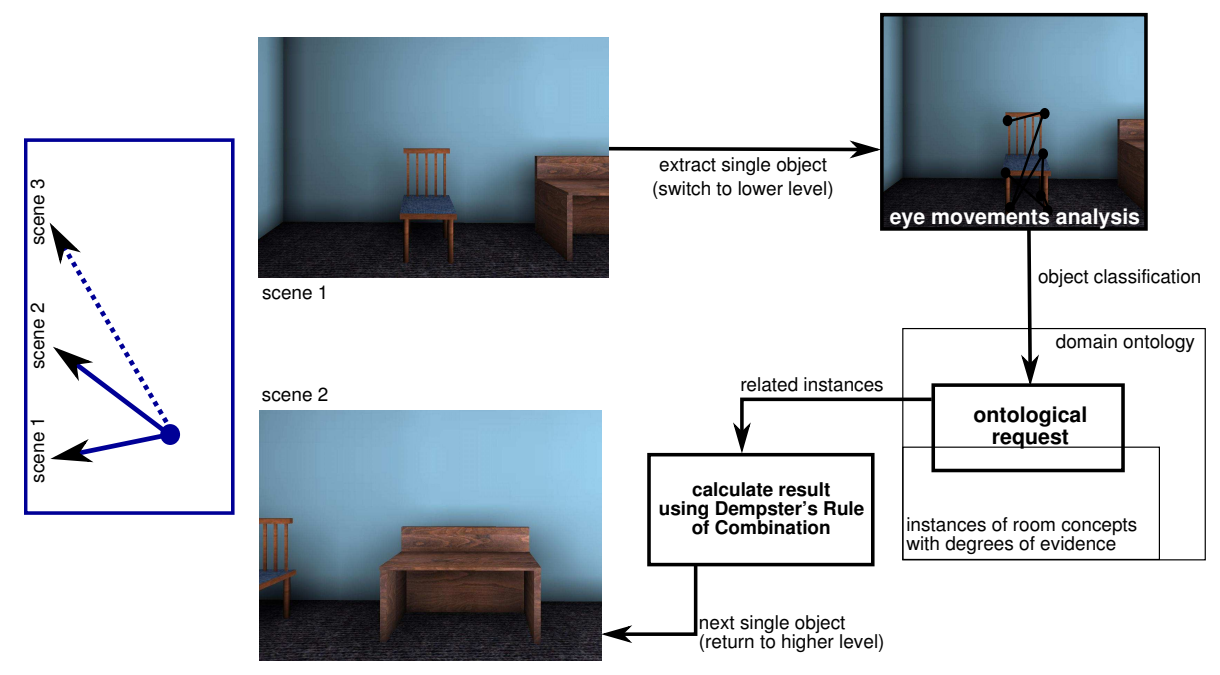

Figure 3: Two-level process of eye movements analysis including the use of ontological and uncertain knowledge

humans tend to recognize single objects and make conclusions about the scene on a more abstract level than pure visual recognition.

For an illustrative test phase, we used a domain of university building rooms. Available room concepts are Kitchen, Classroom, Lecture Hall, Office, and Laboratory. As the system is instructed to identify them from a given input scene with a certain degree of evidence and all room concepts are pairwise distinct in our modelling, the system uses Demster's Rule of Combination of singleton hypotheses as described in [10]. According to the visual analysis of one object after the other, we compute the respective beliefs of each room concept iteratively after every single object classification. This is primarily done for performance, to get a first result of a possible room concept as soon as possible.

The example of a scene analysis that demonstrates the systems behaviour is shown in Figure 4. The physical room scene ${ }^{5}$ is displayed in the upper left of the window, in which arrows indicate performed eye movements on the higher level. Analysed single objects are shown in the main frame, which shows the system's object classification for single objects (compare [17]). Results from domain ontology requests are given at the bottom.

In our example, two specialists have assigned their belief values for prototypical room scenes that support the respective room concepts. Their specifications generally tend to assign more than one room concept. In case of the analysed room scene, shown in the upper left of Figure 4, the average assignments for room concepts are Office (with an evidence of 0.75) and Laboratory (0.05).

First, the system performs eye movements on the higher level on the complete

\footnotetext{
${ }^{5}$ The images have been abstracted from original pictures taken in university buildings for computational simplicity.
} 


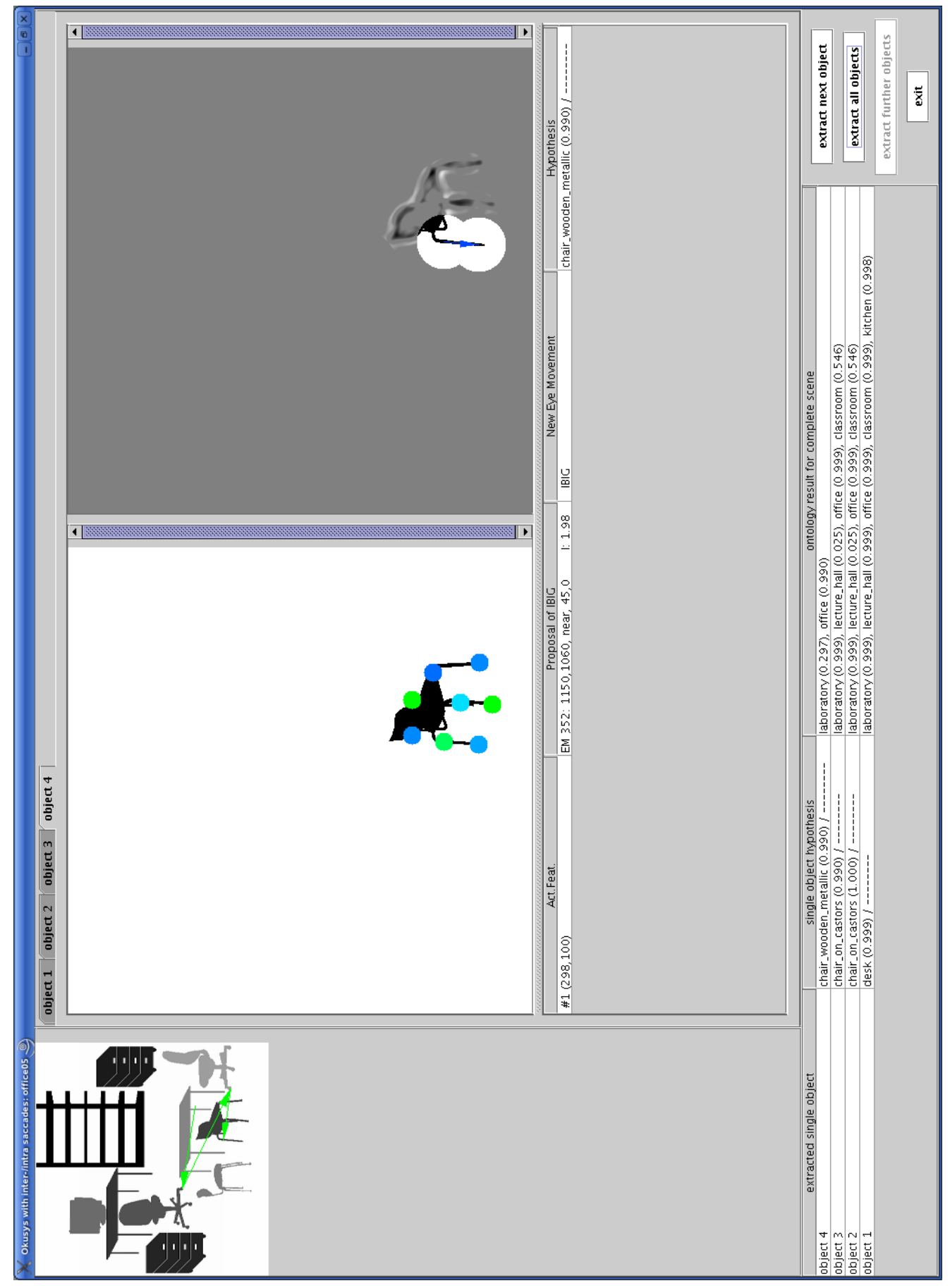

Figure 4: Analysis of room concept with four classified objects 
scene. Beginning at the first fixation, it extracts the object in the image at the corresponding coordinates. This extraction is done on the basis of the respective gray value at this point. Then, the system classifies the extracted object by performing eye movements on the lower level, which is already described in [17]. The result of the classification is passed to the ontology including the request: what kind of room concepts are supported by physical rooms that consists of the (so far) classified objects? In this example, the first classified object was a desk (see Figure 4, fourth row (object 1) of the table at the bottom of the window).

The instances resulting from the specialists' assignments about physical rooms that agree with the analysed constituting object are returned and combined by using Dempsters Rule of Combination as described in section 3. In this case we get the following results for possible room concepts of the current scene given the respective belief values in parentheses: Laboratory (0.999), Lecture Hall (0.999), Office (0.999), Classroom (0.999), Kitchen (0.998). The system continues performing its higher level eye movement and extracts and analyses the object in the image at the next fixation point. In the example shown in Figure 4 the analysis shows a sound result of the room supporting the concepts Office (0.99) and Laboratory (0.297) after four steps with respect to the experimental rating of the specialists.

\section{Conclusions}

In this paper we have introduced a first prototype for integrating uncertain knowledge into ontological knowledge. However, little work has been done on integrating uncertain knowledge into ontologies as yet. Although two approaches tried to combine Bayesian probabilities [6] or Fuzzy Logic [15] with ontologies, both approaches are more interested in the technical issues concerning this combination than their applicability. In this paper we have emphasised the motivation of our decision influenced by the need of a real world application to represent both ontological and uncertain knowledge jointly.

As the structure of our domain ontology evolves from an ontology library we hope to avoid the commonly arising difficulties for coherence, re-use, mapping and extensibility. Still it is up to future work to determine whether the belief values introduced in our domain ontology are also applicable to similar niche concepts. It might also be necessary for consistency to define an additional quality that specifies whether a quality may have several quales with additional evidences.

Also some computational issues will be investigated further: On the one hand, an automatisation for quality concepts that include an evidence quality value, like modeling a "meta-class", will be considered. On the other, the calculation of sets of room concepts for a physical room instead of merely calculating single hypotheses will be integrated as this is one of the major advantages of the Dempster-Shafer theory. 


\section{Acknowledgements}

The Collaborative Research Center for Spatial Cognition (Sonderforschungsbereich Transregio SFB/TR8) of the Universität Bremen and the Universität Freiburg is funded by the Deutsche Forschungsgemeinschaft (DFG), whose support we gratefully acknowledge.

This work has been especially supported by the SFB/TR8 subprojects I1[OntoSpace] and A5-[ActionSpace].

\section{References}

[1] Jeffrey A. Barnett. Computational Methods for a Mathematical Theory of Evidence. In Proceedings of the 7th International Joint Conference on Artificial Intelligence, pages 868-875, Vancouver, Canada, 1981.

[2] John A. Bateman. Linguistic interaction as ontological mediation. In Dietmar Zaefferer and Andrea Schalley, editors, Ontolinguistics. How ontological status shapes the linguistic coding of concepts. Mouton de Gruyter, to appear.

[3] John A. Bateman and Scott Farrar. Spatial Ontology Baseline. SFB/TR8 internal report I1-[OntoSpace] D2, Collaborative Research Center for Spatial Cognition, University of Bremen, University of Freiburg, Germany, June 2005 .

[4] Raj K. Bhatnagar and Laveen N. Kanal. Handling Uncertain Information: A Review of Numeric and Non-Numeric Methods. In Laveen N. Kanal and John F. Lemmer, editors, Uncertainty in Artificial Intelligence, pages 3-26. North-Holland, Amsterdam, 1986.

[5] Tim Burners-Lee, James Hendler, and Ora Lassila. The Semantic Web. Scientific American, 284(5), May 2001.

[6] Zhongli Ding, Yun Peng, and Rong Pan. A Bayesian Approach to Uncertainty Modeling in OWL Ontology. In Proceedings of the International Conference on Advances in Intelligent Systems - Theory and Applications, page 9, Luxemburg, November 2004. IEEE.

[7] Scott Farrar and John A. Bateman. General Ontology Baseline. SFB/TR8 internal report I1-[OntoSpace] D1, Collaborative Research Center for Spatial Cognition, University of Bremen, University of Freiburg, Germany, November 2004.

[8] Scott Farrar, Thora Tenbrink, John A. Bateman, and Robert J. Ross. On the Role of Conceptual and Linguistic Ontologies in Spoken Dialogue Systems. In Symposium on Dialogue Modelling and Generation, Amsterdam, Netherlands, July 2005. 
[9] Asunción Gómez-Pérez, Mariano Fernández-López, and Oscar Corcho. Ontological Engineering with examples from the areas of Knowledge Management, e-Commerce and the Semantic Web. Springer-Verlag, London, 2004.

[10] Jean Gordon and Edward H. Shortliffe. A method for managing evidential reasoning in a hierarchical hypothesis space. Artificial Intelligence, 26:323$357,1985$.

[11] Theo Herrmann. Blickpunkte und blickpunktsequenzen. Sprache und Kognition, 15:159-177, 1996.

[12] Markus Knauff. Räumliches Wissen und Gedächtnis. Deutscher Universitätsverlag, Wiesbaden, 1997.

[13] Claudio Masolo, Stefano Borgo, Aldo Gangemi, Nicola Guarino, and Alessandro Oltramari. Ontologies library (final). WonderWeb Deliverable D18, ISTC-CNR, Padova, Italy, December 2003.

[14] Kevin Mulligan. Perception, Predicates and Particulars. In Denis Fisette, editor, Consciousness and Intentionality: Models and Modalities of Attribution, pages 163-194. Kluwer, Dordrecht, 1999.

[15] Jeff Z. Pan, Giorgos Stamou, Vassilis Tzouvaras, and Ian Horrocks. A Fuzzy Extension of SWRL. In Proceedings of the International Conference on Artificial Neural Networks (ICANN 2005), Special section on "Intelligent multimedia and semantics", 2005. to appear.

[16] Kerstin Schill. Distributed reasoning with uncertain data. In Bilal M. Ayyub and Madan M. Gupta, editors, Uncertainty Analysis in Engineering and the Sciences: Fuzzy Logic, Statistics, and Neural Network Approach, pages 339-351. Kluwer Academic Publisher, Boston, 1997.

[17] Kerstin Schill, Elisabeth Umkehrer, Stephan Beinlich, Gerhard Krieger, and Christoph Zetzsche. Scene analysis with saccadic eye movements: Topdown and bottom-up modeling. Journal of electronic imaging, 10(1):152160, 2001.

[18] Glenn Shafer. A Mathematical Theory of Evidence. Princeton University Press, Princeton, 1976.

[19] Barry Smith and Achille Varzi. The niche. Nô̂s, 33(2):198-222, 1999.

[20] Mike Uschold and Michael Grüninger. Ontologies: Principles, methods and applications. Knowledge Engineering Review, 11:93-155, February 1996.

[21] Alfred L. Yarbus. Eye Movements and Vision. Plenum Press, New York, 1967. Translation: B. Haigh. 